\title{
Increased serum levels of the Wnt antagonist Dickkopf-1 (DKK1) and impaired trabecular bone mineral density using QCT scan in acromegalic patients
}

Elena Valassif ${ }^{1}$, Iris Crespo ${ }^{1}$, Anna Aulinas ${ }^{1}$, Eulalia Urgell2, Jorge Malouff, Jaume Llauger ${ }^{4}$, Ana Maria Marin ${ }^{3}$, Betina Biagetti, ${ }^{5}$ Susan M. Webb'

${ }_{1}^{1}$ IB-Sant Pau and Department of Endocrinology/Medicine, Hospital Sant Pau, UAB and Centro de Investigación Biomédica en Red de

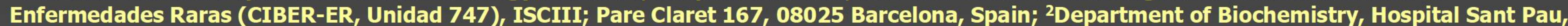
Barcelona (Spain) ${ }^{3}$ Department of Internal Medicine, Bone Mineral Metabolism Unit, Hospital Sant Pau, Barcelona (Spain); ${ }^{4}$ Department of Radiology, Hospital Sant Pau, Barcelona (Spain); ${ }^{5}$ Endocrinology Department, Hospital Vall d'Hebron

\section{OBJECTIVES}

- To assess volumetric bone mineral density (VBMD) in patients with acromegaly.

\section{METHODS}

- Thirty-one acromegalic patients [17 (55\%) men; 18 (58\%) with active disease; mean age $48.2 \pm 7.5$ years (range $28-65$ years)] and thirty-two age-, gender- and BMI-matched controls.

- Volumetric QCT acquisitions of the proximal hip were performed. (Philips Brilliance CT 16-slice). All the QCT data were processed using QCT-pro Bone Investigational Toolkit Version 2.0 (BIT, Mindways).
To compare vBMD in patients with acromegaly vs. age-, gender-, and BMI-matched controls.

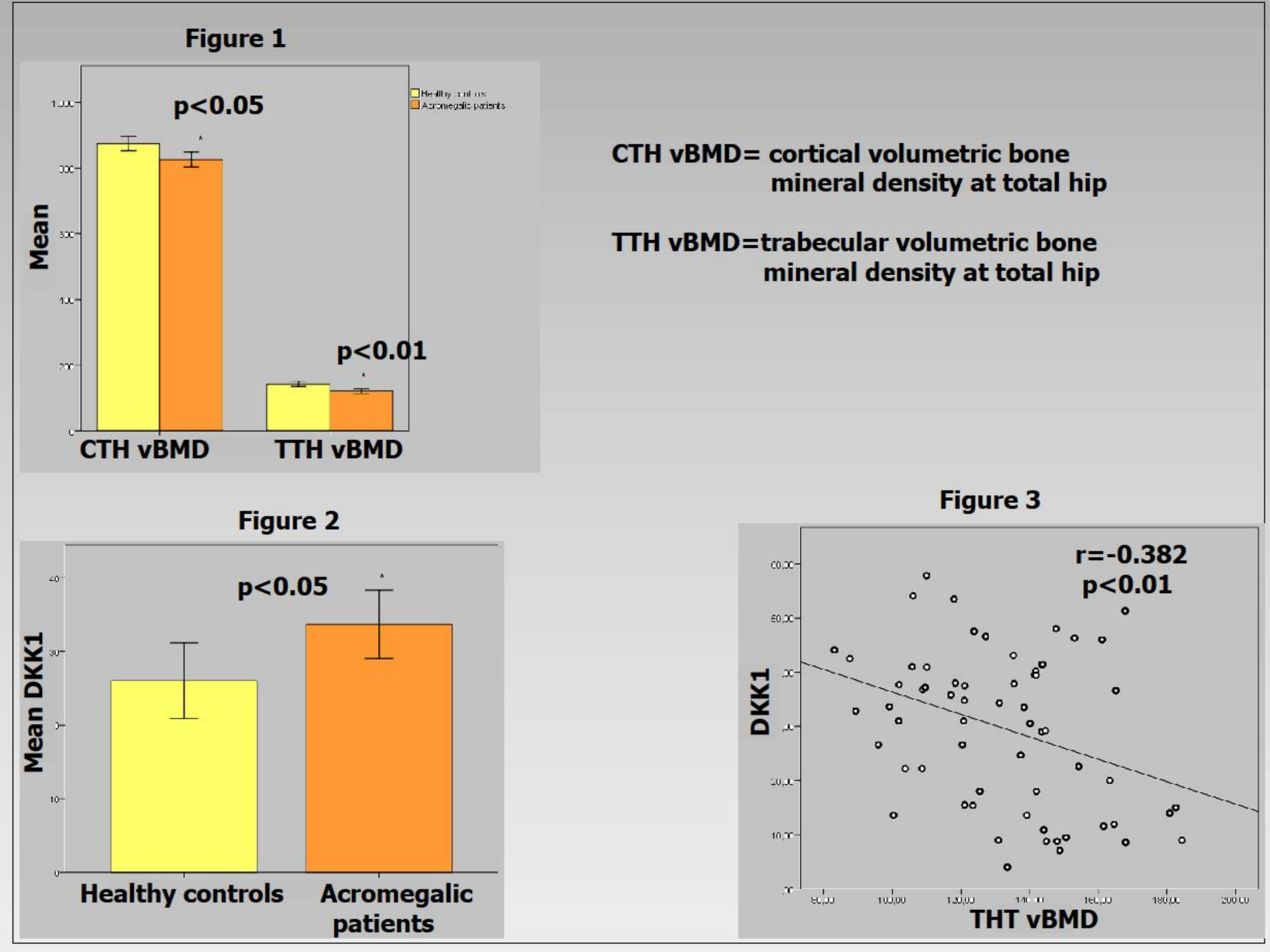

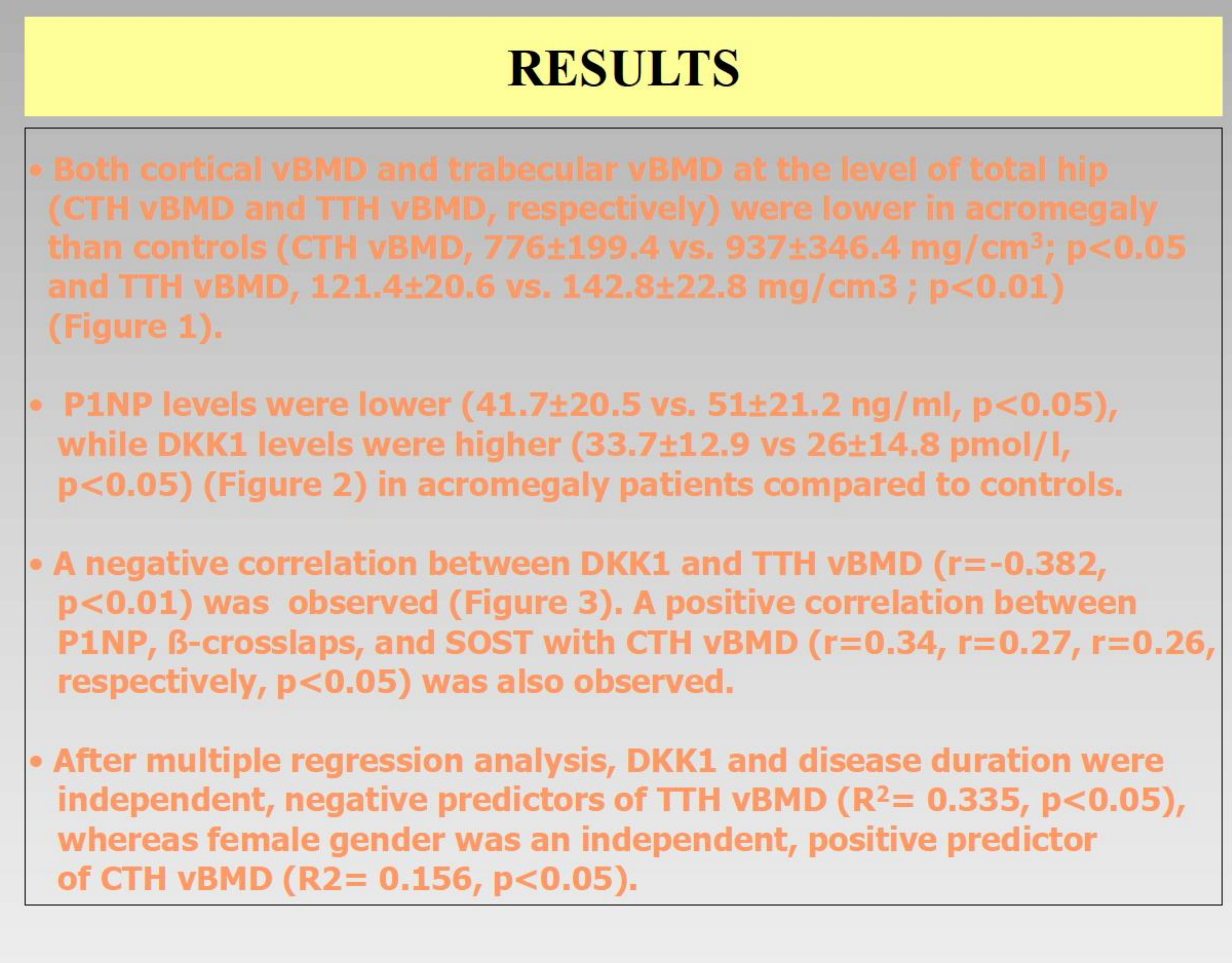

REFERENCES:

- Sclerostin and dickkopf-1 as therapeutic targets in bone diseases. Endocrine Rev 2012; 33:747-783

total hip compared with healthy controls.

- The Wnt signaling antagonist DKK1 may contribute to the skeletal fragility described in acromegaly.
- Mazziotti G, Biagioli E, Maffezzoni F et al. Bone acromegaly: a meta--analysis. J Clin Endocrinol Metab 2015; 100:384-394 turnover, bone mineral density, and fracture risk in 